\title{
Interview
}

\section{Antibiotika-Resistenzen: Die Politik redet, Ärzte handeln}

Die Politik hat sich 2015 erstmals groß dem Thema Antibiotika-Resistenzen gewidmet. Aber hatte das auch Auswirkungen auf den Praxisalltag? Und wie gehen einzelne Fachgruppen das Problem an? Springer Medizin hat vier Kollegen aus vier Disziplinen gefragt. Die vier Interviews können Sie auf unserer Homepage sehen (s. Kasten). Für die HNO hat Prof. Ludger Klimek unsere Fragen beantwortet.

? Die große Politik hat sich in den vergangenen Monaten intensiv mit dem Thema Antibiotika-Resistenz befasst. Gröhes 10-Punkte-Plan, DART 2020, G7-Gipfel - was ist davon in Ihrer Praxis angekommen?

Prof. Dr. med. Ludger Klimek: Nun, davon sicherlich noch relativ wenig. Die Überlegungen, Antibiotika möglichst gezielt und sinnvoll einzusetzen, bestanden eigentlich schon vorher. Dass die politischen Vorgaben im Moment etwas daran geändert hätten, kann ich eigentlich nicht sagen.

? Die Politik redet, Ärzte müssen handeln - wie gehen Sie bei der Therapie von Atemwegsinfekten vor, um der Entstehung von Antibiotika-Resistenzen entgegenzuwirken? Prof. Klimek: Antibiotika sollten generell nur sehr gezielt eingesetzt und nicht vorschnell verordnet werden. Aber wenn sie gebraucht werden, dann müssen sie so gezielt und auch konsequent eingesetzt werden - also auch ausreichend langfristig -, dass sie die gewünschte Wirksamkeit haben. Wenn wir das so machen, sind Antibiotika mit die wertvollsten Medikamente, die wir haben. Das Entscheidende ist der gezielte, sinnvolle Einsatz.

? Welchen Stellenwert haben Phytopharmaka in Ihrer Praxis bei der Behandlung von Atemwegsinfekten?

Prof. Klimek: Nun, einen riesengroßen - der ist gar nicht zu überschätzen. Phytopharmaka gehören für mich bei den akuten Atemwegsinfekten zu den Therapeutika der ersten Wahl - in unterschiedlicher Ausprägung und unterschiedlicher Zielrichtung. Aber definitiv sind es wahrscheinlich auch die wichtigsten Präparate überhaupt.

? Wenn Sie Ihre eigene Fachgruppe betrachten im Hinblick auf deren Rolle beim Trend hin zum rationalen Einsatz von Antibiotika, wo sehen Sie die HNOÄrzte: Vorreiter oder Schlusslicht?

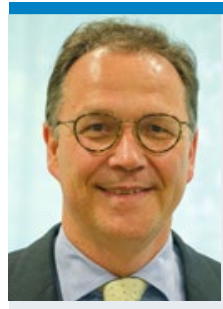

\section{„Bei Atemwegsinfekten können Antibiotika oft vermieden werden. HNO-Ärzten kommt deshalb hinsichtlich der Entscheidung für oder wider Antibiotika eine Vorreiterrolle zu."}

Prof. Dr. Ludger Klimek

ist Facharzt für HNO-Heilkunde am Zentrum für Rhinologie und Allergologie in Wiesbaden

Prof. Klimek: Die HNO-Ärzte haben eine absolute Vorreiterrolle, weil sie häufig die Patienten sehen, bei denen Antibiotika vermieden werden können. Sie sind aber auch diejenigen, die entscheiden müssen, wann Komplikationen drohen, wann Antibiotika eingesetzt werden müssen. Das heißt, die Entscheidung für oder wider Antibiotika bei Infekten der oberen Atemwege, bei Erkrankungen der Nase und der Nasennebenhöhlen können eigentlich die HNO-Ärzte am besten treffen.

! Das Interview führte Andrea Wehrens.

\section{Interviews im Video}

Unter http://tinyurl.com/zcmz6kj können Sie das Interview mit Prof. Klimek als Video sehen. Dort finden Sie auch die Interviews mit den Kollegen aus der der Inneren Medizin, der Pädiatrie und der Allgemeinmedizin.

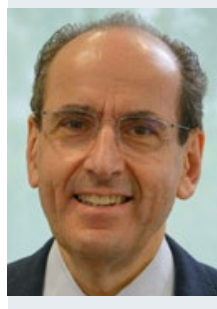

\section{Dr. Peter Kardos}

Facharzt für Innere Medizin, Schwerpunkt Pneumologie, Frankfurt am Main

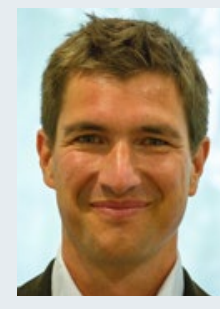

\section{Dr. Daniel Seng}

Facharzt für Kinderund Jugendmedizin, Unterschleißheim bei München

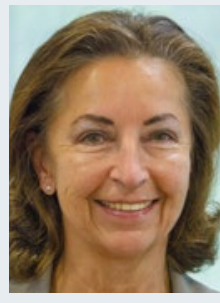

Dr. Margit Hollenz

Fachärztin für Allgemeinmedizin, Rödental 